\title{
A COMPARATIVE STUDY OF MAGNETIC RESONANCE IMAGING, ELECTRICAL IMPEDANCE TOMOGRAPHY AND ULTRASONIC DOPPLER VELOCIMETRY FOR SEMI-DILUTE FIBRE FLOW SUSPENSION CHARACTERISATION
}

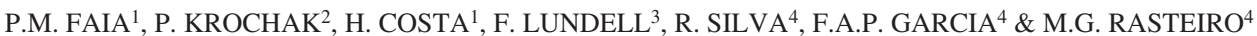 \\ ${ }^{1}$ Electrical and Computers Engineering Department, Faculty of Sciences and Technology of the University of \\ Coimbra and CEMUC - Centre of Mechanical Engineering, Portugal. \\ ${ }^{2}$ Innventia $\mathrm{AB}$, Sweden. \\ ${ }^{3}$ Wallenberg Wood Science Centre, Royal Institute of Technology, Sweden. \\ ${ }^{4}$ Chemical Engineering Department, Faculty of Sciences and Technology of the University of Coimbra, and \\ CIEPQPF - Research Centre on Chemical Process and Forest Products, Portugal.
}

\begin{abstract}
Experimental comparisons between imaging techniques serve to provide confidence in the validity of each technique for the study of multiphase flow systems. Such cross-validation can establish the limitations of each technique quantitatively. In the present paper, the authors report efforts made on the characterization of semi-dilute, mono-dispersed suspensions of rayon fibres in turbulent water flow using Magnetic Resonance Imaging (MRI), Ultrasound Velocity Profiling (UVP) and Electrical Impedance Tomography (EIT). Increasing flow velocities and fibre concentration were studied using these three experimental techniques. For lower fibre concentrations more uniform distributions were observed and as flow velocity increased fibre agglomerations were found in the centre region of the pipe.

Keywords: EIT, industrial tomography, MRI, semi-dilute fibre suspensions, spatial distribution of phases, UVP.
\end{abstract}

\section{INTRODUCTION}

In the development of accurate models for processes where suspensions are present, prior knowledge of several flow characteristics is essential, such as spatial distribution of phases, flow regimes, relative velocity between phases, etc. Several non-invasive techniques of flow characterization and examples of their application can be found in the literature [1,2].

Magnetic Resonance Imaging (MRI) is a tomographic technology with a high spatial resolution that can supply information about the behaviour of protons present in a system, usually contained in the $1 \mathrm{H}$ nuclei of water. MRI can be used, amongst other things, to image motion of water, e.g. expressed in the form of velocity profiles. The major limitations of MRI are the types of particles and the size of the system that can be studied, together with the size, weight and cost of the instrument. Only particles containing MR-sensitive nuclei, such as $1 \mathrm{H}$, can be detected $[3,4]$. The maximum diameter of the system is bounded by the inner diameter of the coil in the magnet. Thus, MRI experiments are typically limited to laboratory-scale fluidised beds or small diameter pipes [5]. In summary, the strength of MRI 
is in studying centimetre-scale systems at spatial resolutions of $\sim 100 \mu \mathrm{m}$. Electrical Impedance Tomography (EIT) can be used to study much larger-scale systems and gives good quality qualitative images of gas-liquid-solid distributions [6]. Among others, some of the advantages of EIT are its portability, simplicity of scale up, and relative low cost. Spatial resolution, however, is typically limited: this is due to both the reconstruction algorithms used (the nature of the reconstruction problem posed by EIT, which is ill conditioned and non-linear, makes the detection of small objects a challenge) and the number of unique measurements of conductivity obtainable, as derived from the current injection-voltage measurement system (very strongly dependent on the number of measuring electrodes) [7]. Furthermore, information concerning the absolute amount of solids or fibres suspended, cannot be accurately determined, since the images obtained represent not absolute values of the conductance/impedance, but the distribution of their variation. The measurement of instantaneous velocities in water flows has long been a challenging issue. Due to their relatively low price and easy handling, Acoustic Doppler systems are widely used at present. Ultrasound systems, based on echography and Doppler Effect, allowed the development of equipment capable of measuring almost instantaneous velocity profiles [8]. Initially, the Ultrasonic Velocity Profile (UVP) technique was limited to opaque fluids [9] and it was typically used to measure across pipe walls in small scale systems [10]. A UVP probe emits an ultrasound beam that travels along the axis and afterwards receives the echo from the same beam as reflected by small particles present in the fluid. The UVP system measures the time delay of the echo to reach the probe and the Doppler frequency shift. Knowing the speed of the sound in the fluid, it calculates the distance and the velocity of the particle in the direction of the beam. Although the UVP technique has been developed for 1D measurements, measurement of $2 \mathrm{D}$ velocity fields using UVP probes have been reported to give promising results [8].

Experimental comparison between imaging techniques serve to provide confidence in the validity of each technique for the study of multiphase flow systems. Such cross-validation can establish the limitations of each technique quantitatively and are necessary to improve imaging methods. Some comparative studies can be found in the literature [11, 12]: however, none of them reports the study of fibre suspension flow using simultaneously UVP, MRI and EIT, at least not to the best of the author's knowledge. In the present paper, the authors report efforts made on the characterization of semi-dilute, mono-dispersed suspensions of rayon fibres in turbulent flow, with increasing speeds and concentrations, in a pilot rig at a laboratorial scale, using both MRI, EIT and UVP. Direct comparisons of EIT, MRI and UVP measurements acquired are presented and the level of agreement explored.

\section{EXPERIMENTAL SETUP}

\subsection{Flow loop}

The laboratory experiments were performed in the joint re-circulatory pipe flow facility of KTH Royal Institute of Technology and Innventia AB in Stockholm Sweden. A scheme of the rig exhibiting the location of all of the experimental sensors can be seen in Fig. 1.

The main part of this facility consists of a section of cylindrical Perspex pipe, with length $7.0 \mathrm{~m}$ and inner diameter $50 \mathrm{~mm}$. The fluid is re-circulated from an open reservoir, approximately 100 litres in volume. The bulk suspension flow is driven by an ITT $2.0 \mathrm{~kW}$ variable frequency drive (ABB ACS 550) centrifugal pump (Flygt pump 3,102-135 mm impeller). 


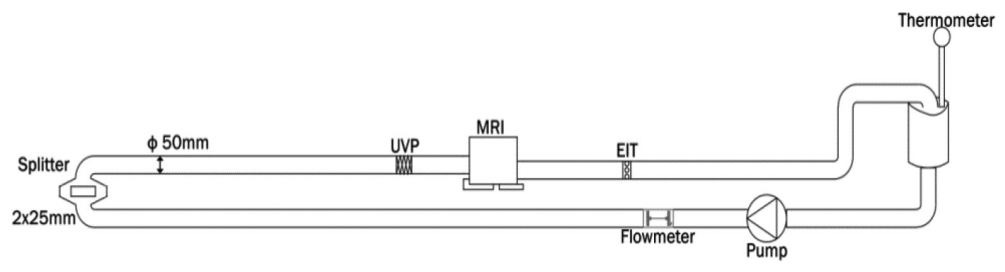

Figure 1: Flow loop schematic.

The flowrate is measured with an ABB magnetic flow meter (Fischer \& Porter model no. 10DS3111). The flow is diverged and then re-converged with a flow splitter positioned at the upstream end of the test section. This minimizes any effects such as swirl resulting from the approach flow to the test section. The UVP measurement section is located $3.5 \mathrm{~m}$ downstream of the flow-splitter, the MRI measurement section is located $4.7 \mathrm{~m}$ downstream the flow splitter or an equivalent 94 pipe diameters and the EIT is mounted at an additional 1.0 $m$ downstream of the MRI. MRI measurements are made in both the vertical and horizontal planes while UVP measurements are collected along the horizontal plane only. The MRI system consists of a 1 Tesla permanent magnet connected to a Bruker NMR spectrometer. A $60 \mathrm{~mm}$ RF coil, calibrated to $43.5 \mathrm{MHz}$ is used for transmission/reception. The entire system has been provided by Aspect Imaging and is controlled using NTNMR software. Devoted measurement and data processing software was developed at the University of California Davis and further adapted in-house for post-processing. Regarding UVP, and for the present experiments, a $4 \mathrm{MHz}$ transducer with a $5 \mathrm{~mm}$ active diameter element (maximum resolution of $0.37 \mathrm{~mm}$ ) and a minimum measuring distance (near field) of $16.9 \mathrm{~mm}$ was used. After this minimum, the beam diverges with a half-angle of $2.2^{\circ}$. The pulse repetition frequency was $10.762 \mathrm{kHz}$ with 128 spatial measurement channels. The data were acquired over 768 measurement cycles to obtain the velocity distribution profile, and mean data was obtained by averaging over 64 profiles. This gives spatial and velocity resolutions of $0.37 \mathrm{~mm}$ and $3.8 \mathrm{~mm} / \mathrm{s}$, respectively. The transducer was flush mounted to the inside pipe wall at a $70^{\circ}$ angle to the flow and was in direct contact with the suspension. With this approach, the effects of attenuation and wall reflections were reduced. The UVP hardware was provided by MetFlow and the software, FlowViz, was developed by SIK in Gothenburg, Sweden. The EIT system electronics and control/operation software developed and used by the authors in the present work have already been described in a previously published work [13]: it measures the differences in both the real and imaginary parts of the impedance, so no data are lost. For the depicted tests an EIT test section with 16 titanium electrodes was produced: each electrode has a diameter of $5 \mathrm{~mm}$ which were equally spaced around the test section perimeter. In all the tests, an excitation frequency of $10 \mathrm{kHz}$ with $2 \mathrm{Vpp}$ amplitude was used: opposite injection and adjacent measuring protocol was used (water conductivity was initially set to $1,600 \mu \mathrm{S} / \mathrm{cm}$ ). For image reconstruction, the open source software EIDORS [14], considering direct differential measured voltages and using a structured mesh consisting of 2,304 linear elements and 1,201 nodes was used (in the reconstructed images the electrode corresponding to higher vertical position is electrode number 1). This software implements a non-linear back projection method using a regularization algorithm (Tikhonov's regularization). To solve the forward problem the Complete Electrode model (CEM) [15] was chosen: this model incorporates the shunt effect and the contact impedance in the electrode/domain interface. 
Table 1: Concentrations and flow velocities tested.

\begin{tabular}{|c|c|c|c|}
\hline Concentrations & $\begin{array}{l}\text { Total mass of } \\
\text { suspended fibres (gr) }\end{array}$ & Flow velocities (m/s) & $\begin{array}{l}\text { Corresponding } \\
\text { Reynolds number }\end{array}$ \\
\hline $\mathrm{nL}^{3}=5$ & 500 & $0.5,1,1.5$ & $25,000,50,000,75,000$ \\
\hline $\mathrm{nL}^{3}=13$ & 1,300 & $0.5,1,1.5$ & $25,000,50,000,75,000$ \\
\hline $\mathrm{nL}^{3}=20$ & 2,000 & $0.5,1,1.5$ & $25,000,50,000,75,000$ \\
\hline
\end{tabular}

2.2 Fibre suspensions and measurement conditions

The suspension consisted of rayon fibres, with mean length, $\mathrm{Lf}=2.0 \mathrm{~mm}$, mean diameter, $\mathrm{df}$ $=60 \mu \mathrm{m}$, and specific gravity, SGf $=1.50$, suspended in tap water. The aspect ratio of these fibres was, $\mathrm{rf} \approx 33$. Among those tested, three different dimensionless concentrations were considered for discussion, namely $\mathrm{nL}^{3}=5,13$ and 20 , where $\mathrm{n}$ is the number of fibres per unit volume of suspension. These concentrations lie in the semi-dilute range, i.e. $1 \leq n L^{3} \leq r_{f}$. Measurement conditions are summarized in Table 1

\section{RESULTS AND DISCUSSION}

As stated, the main objective of the current work is to present direct comparisons between EIT, MRI and UVP measurements. For that purpose, the authors start by discussing the acquired velocity profiles using UVP and MRI, followed by interpretation of the obtained 2D cross-section conductivity distribution maps using EIT. They will conclude this discussion section giving their interpretation of the overall presented results.

\subsection{MRI vs. UVP}

Along this section, where MRI and UVP results are presented and discussed, the horizontal velocity profiles obtained using MRI, are indicated in the plots by $\mathrm{MRI}_{\mathrm{H}}$, while the data obtained by each independent UVP probe are indicated as $\mathrm{UVP}_{4}$ and $\mathrm{UVP}_{9}$, which refers to their respective hardware channel. Velocity data is used from each probe up to the channel centreline. In the cases where MRI and UVP are directly compared on the same plot, we use only the horizontal MRI measurement since the UVP probes were positioned horizontally with respect to the pipe. In Fig. 2, measurements with dilute suspensions, i.e. $\mathrm{nL}^{3}=5$, are shown. In these, and all subsequent plots, the UVP measurements are made along the horizontal plane, and the MRI along both horizontal and vertical planes. Excellent agreement between the two measurement techniques can be observed. Moreover, both the UVP and MRI velocity profiles are noted to be symmetric about the pipe axis (for the range of UVP validity). These results show that, since the fibres are assumed to translate with the carrier fluid (without altering the flow structure itself) at dilute concentrations, the measurement of fibre motion with UVP should agree with that for water measured with MRI. Figure 2e and $\mathrm{f}$ show the velocity profiles obtained using MRI for $\mathrm{nL}^{3}=5$ in both vertical and horizontal planes, respectively, for increasing velocities. Figure $2 \mathrm{~d}$ shows the same measurements made with UVP: no change in the UVP velocity profiles can be observed as velocity increases. However, slight changes are observed with the MRI, namely the profiles become slightly broader, i.e. more blunt-like as the velocity is increased. It should also be noted that a slight difference is observed between the horizontal and vertical slices. Figure 3 compares the 

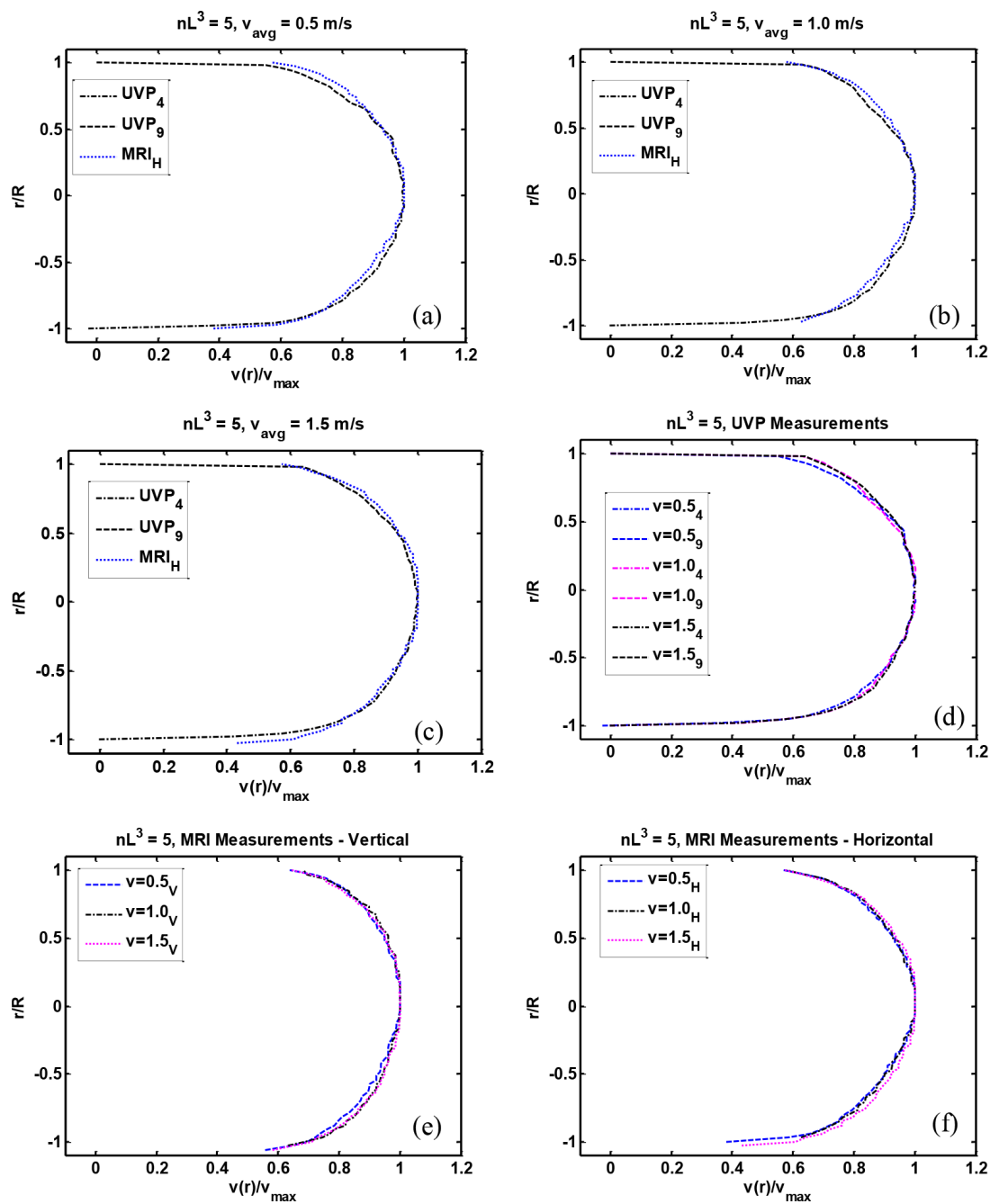

Figure 2: Velocity influence on fibre flow behaviour for a fixed concentration of $\mathrm{nL}^{3}=5$ : (a) $0.5 \mathrm{~m} / \mathrm{s}$; (b) $\mathrm{v}=1 \mathrm{~m} / \mathrm{s}$; (c) $\mathrm{v}=1.5 \mathrm{~m} / \mathrm{s}$; (d) UVP; (e) vertical MRI; (f) horizontal MRI.

profiles measured with UVP and MRI and the effect of increasing velocity for $\mathrm{nL}^{3}=13$ and $\mathrm{nL}^{3}=20$. Here, we see that for both concentrations, the UVP profiles are essentially unaffected by the velocity increase. The MRI profiles, however, show some subtle differences: a small difference between the horizontal and vertical velocity profiles is perceptible. This could be due to the design of the flow loop and possibly some residual effects of the entry region, despite the fact that we are measuring nearly 100 pipe diameters downstream. Still the velocity profiles are symmetric. It may also be seen that as the velocity is increased, slight differences between water phase velocity (measured with MRI) and fibre phase velocity (measured with UVP) exist. From the same figures we can compare the profiles measured with UVP and MRI and the effect of increasing concentration for mean velocities of $0.5,1.0$ and $1.5 \mathrm{~m} / \mathrm{s}$. 

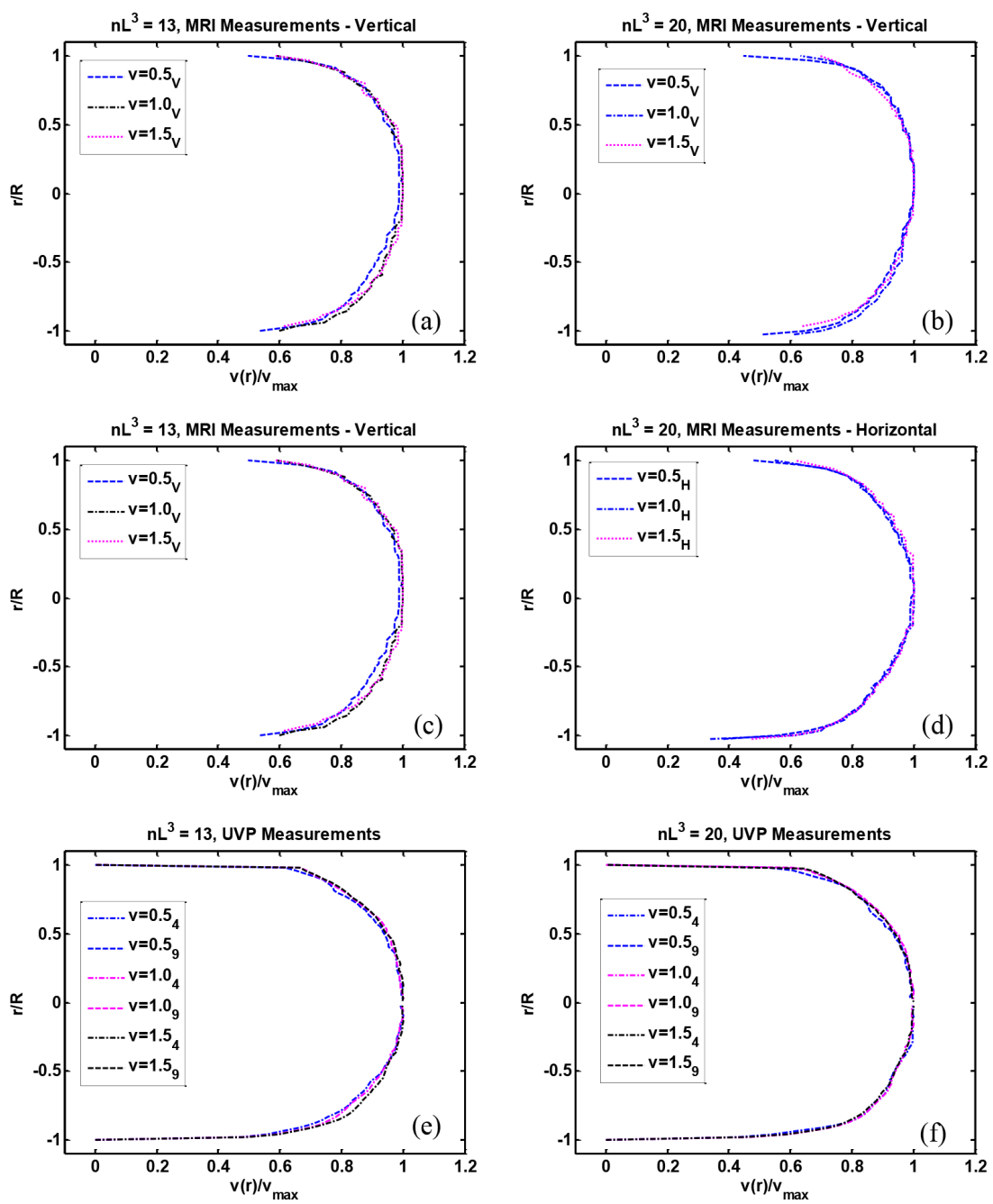

Figure 3: Velocity influence on fibre flow behaviour for fixed concentrations of $\mathrm{nL}^{3}=13$, (a) vertical MRI, (c) horizontal MRI, (e) UVP, and for $\mathrm{nL}^{3}=20$, (b) vertical MRI; (d) horizontal MRI; (f) UVP.

For lower velocities, i.e. 0.5 and $1.0 \mathrm{~m} / \mathrm{s}$, we see a clear effect of increased concentration on both the MRI and UVP profiles. Specifically, both profiles become more flat (top hat like) as the concentration increases. This can be due to increased fibre flocculation. At the highest velocity, i.e. $1.5 \mathrm{~m} / \mathrm{s}$, the velocity profiles measured with UVP are essentially unaffected by the change in concentration. This may be explained by the increased inertia in the suspension at high velocity (Reynolds number) and also by increased fluidization of the suspension due to turbulence and shear. One can also observe a larger difference in the MRI velocity profiles as the concentration is increased compared with changes in the UVP profiles. This again could be an indication of very small differences between the water and fibre phases. 


\subsection{EIT}

In this section, the authors present and analyse the conductivity distributions of the pipe flow cross sections obtained. In the EIT images, a darker blue colour corresponds to a lower conductivity region, which is due to the presence of a higher quantity of fibres in that same area of the pipe section: in opposition, yellow or red colours identify higher conductivities regions, corresponding to the presence of a much lower quantity of fibres in that same area of the pipe section. From Figs 4 and 5, the changes in the distribution of fibres in the pipe cross section are observed, with increasing velocity, for different fibre concentrations (colour bar units is $\mu \mathrm{S} / \mathrm{cm})$. For the lowest fibre concentration, at the lower velocity, the fibres are very dispersed in the tube section (Fig. 4a): the impedance distribution visible in the centre of the image exhibits a higher conductivity region due to a very low quantity of fibres which are highly dispersed. As velocity is raised, the fibres begin to agglomerate in the centre of the pipe section (confirmed by the more uniform blue regions in the centre of the section in Fig. 4b and $\mathrm{c}$, which is due to the large quantity of fibres present in that area of the pipe).

For the second presented concentration, of $\mathrm{nL}^{3}=13$, the quantity of suspended fibres is much higher when compared with the first concentration of $\mathrm{nL}^{3}=5$, but at the lowest velocity the fibres are still very distributed along the pipe section (Fig. 5a).The effect of the velocity is only visible when it is further raised: indeed, when the velocity is increased to $1 \mathrm{~m} / \mathrm{s}$ and then to $1.5 \mathrm{~m} / \mathrm{s}$, the fibres begin to agglomerate in the centre of the pipe section (confirmed by the more uniform, but increasing smaller and darker blue region in the centre of the pipe section in Fig. 5c and e). For the highest fibre concentration, the effect of velocity is more enhanced: the fibres start to be apparently uniformly dispersed (Fig. 5b); then, due to the velocity increase they are forced to be agglomerated at the centre of the pipe, at the intermediate velocity of $1 \mathrm{~m} / \mathrm{s}$ (Fig. 5d). However, at the highest velocity, the obtained result is not coherent with what would be expected and with what was observed for the previous

(a)

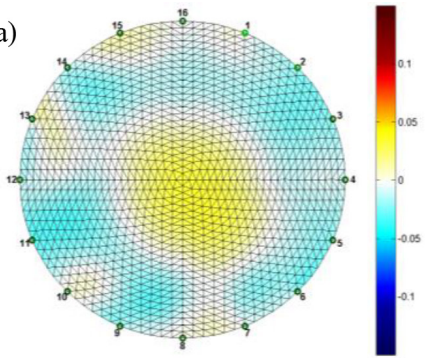

(b)

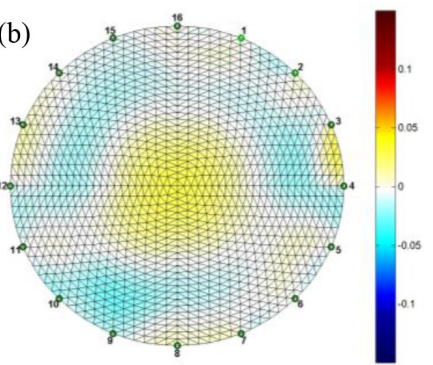

(c)

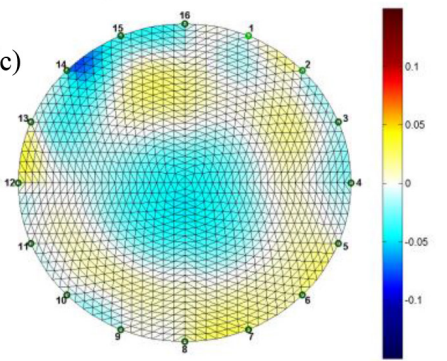

Figure 4: Velocity influence on fibres flow behaviour for a fixed concentration of $n L^{3}=5$ : (a) $0.5 \mathrm{~m} / \mathrm{s}$; (b) $1 \mathrm{~m} / \mathrm{s}$; (c) $1.5 \mathrm{~m} / \mathrm{s}$. 
concentrations: in fact, it is not observable with the velocity increase a further increment of fibres concentration in the centre region of the pipe section (Fig. 5f). From the same figures the effect of fibre distribution with concentration increase for fixed velocities can be observed. For the lowest velocity and lowest concentration, the volume of fibres is low and, consequently, the impedance distribution visible in the centre of the image, for this velocity, exhibits a higher conductivity, consequence of a very high dispersion along the pipe section. As the concentration of the fibres is increased, the impedance distribution becomes more uniform: in fact, the blue region in the images, identifying the lower conductivity area which is a synonym of the presence of a larger quantity of fibres in that area of the pipe, becomes larger and more uniformly distributed. At $1 \mathrm{~m} / \mathrm{s}$, for the lowest concentration, since the volume of fibres is low, the low impedance area visible in the centre of the images is still noticeable. However, its intensity, identifiable by a much lighter yellow and much smaller occupied area in the image, reveals that the fibres dispersion is not has high as it was for the lowest velocity of $0.5 \mathrm{~m} / \mathrm{s}$. As concentration increases, the effect of velocity increase is noticeable. Indeed, the fibres become more and more agglomerated in the centre of the pipe

(a)

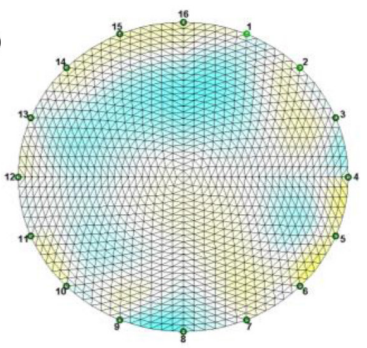

(c)

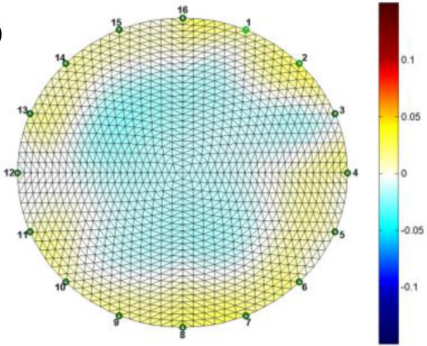

(e)

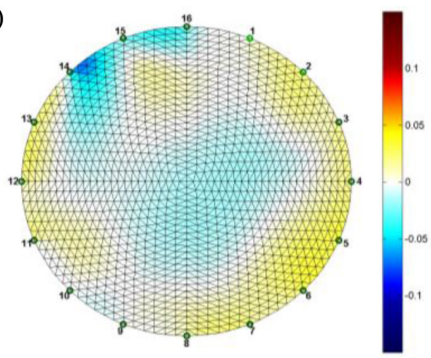

(b)

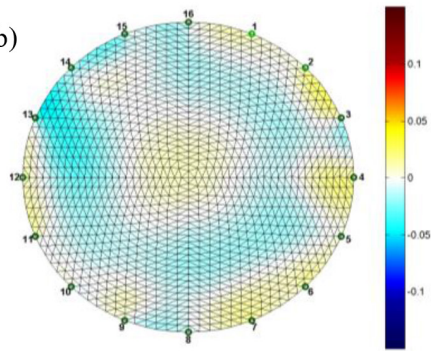

(d)

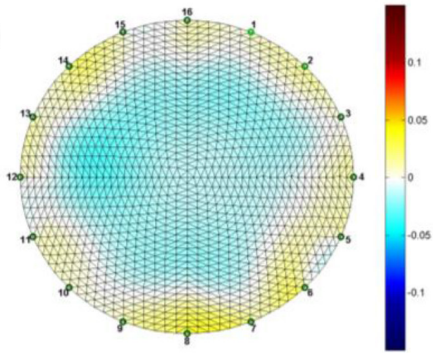

(f)

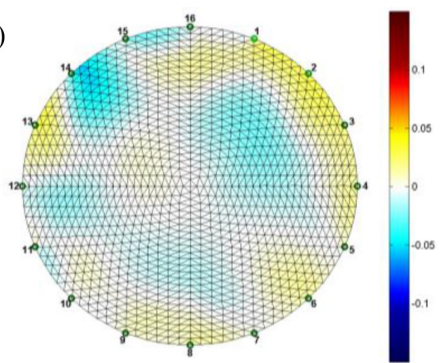

Figure 5: Velocity influence on fibres' flow behaviour for fixed concentrations of $\mathrm{nL}^{3}=13$, (a) $0.5 \mathrm{~m} / \mathrm{s}$, (c) $1 \mathrm{~m} / \mathrm{s}$, (e) $1.5 \mathrm{~m} / \mathrm{s}$, and of $\mathrm{nL}^{3}=20$, (b) $0.5 \mathrm{~m} / \mathrm{s}$; (d) $1 \mathrm{~m} / \mathrm{s}$; (f) $1.5 \mathrm{~m} / \mathrm{s}$. 
section (the blue region in the images, identifying the lower conductivity area, becomes larger and more intense indicating the presence of an increasing number of fibres in those areas of the pipe section). At the highest velocity, for the lowest fibre concentration, the fibres are highly agglomerated in the centre of the pipe (the darker blue region in the pipe centre is much smaller and less intense). With fibre concentration increment, a larger blue region is visible, with the exception of the incoherence, already mentioned, found for the highest velocity and concentration presented, where a larger blue region in the centre of the pipe was expected compared with the intermediate concentration.

\subsection{Combined experimental techniques analysis}

Correlations can be observed between EIT and MRI/UVP regarding the effect of concentration increase at lower velocities, i.e. $0.5 \mathrm{~m} / \mathrm{s}$ and $1.0 \mathrm{~m} / \mathrm{s}$ : specifically, with both MRI and UVP, the velocity profiles become more flat. The EIT measurements at these velocities, indicate that the fibres are becoming more spread out within the pipe. Thus we can correlate an increase in the velocity profile bluntness and the fibre distribution in the channel. For instance, at the velocity of $1 \mathrm{~m} / \mathrm{s}$ in the MRI profiles, slight changes can be seen with the fibre concentration increase: profiles become slightly broader, which is a consequence of a larger quantity of fibres. Meanwhile, at the same velocity, in the EIT images we can observe very similar phenomena: as concentration increases, the blue region in the images, identifying the lower conductivity area, becomes larger and more intense indicating the presence of an increasing number of fibres in those areas of the pipe section. Similar correlations can be pointed out when fixing a certain concentration, while flow velocity increases. In fact for $\mathrm{nL}^{3}=13$, the MRI profiles show that as the velocity increases, the velocity profile gets thicker (see the different between $1 \mathrm{~m} / \mathrm{s}$, and $1.5 \mathrm{~m} / \mathrm{s}$ ). Comparable observations can be made by looking at EIT images: at the lowest velocity, the fibres are still very distributed along the pipe section, then, when velocity is further raised to $1 \mathrm{~m} / \mathrm{s}$ and then to $1.5 \mathrm{~m} / \mathrm{s}$, the fibres begin to increasingly agglomerate in the centre of the pipe section. Based on this, the authors believe that not only the three experimental techniques provide very important information regarding the characterisation of fibre suspension flow, as their combined use enriches and strengthens the conclusions about the way the fibre suspensions behave, depending on concentration and velocity changes.

\section{CONCLUSIONS}

In the present paper, the authors have presented results about the characterization of semidilute, mono-dispersed suspensions of rayon fibres in turbulent flow, with increasing velocities and concentrations, in a pilot rig at a laboratorial scale, using both MRI, EIT and UVP. Discussion of the results have shown that the comparison of the results between EIT, MRI and UVP measurements presented a great level of agreement, indicating that the simultaneous future use of these techniques will allow a better knowledge of the suspensions flow behaviour. In particular, the possibility to compare pilot and lab scale measurements with all three techniques and plant scale measurements with one or two is a promising possibility. Here, correlations between measuring techniques were made for increasing concentrations and increasing velocities. So, in conclusion, the combined use of these three techniques supplies a better insight about the way the fibre suspensions behave as a function of concentration and velocity changes. Even though the changes detected in the present ranges $\left(0<\mathrm{nL}^{3}<20\right.$ and flow velocities between 0.5 and $1.5 \mathrm{~m} / \mathrm{s}$ ) were moderate, they could still be detected in a consistent manner with all three techniques. 


\section{ACKNOWLEDGEMENTS}

COST Action FP1005 support, fibre suspension flow modelling - a key for innovation and competitiveness in the pulp and paper industry - is gratefully acknowledged. The authors all thank the support of Fundação para a Ciência e Tecnologia through their projects, contract PTDC/EQU-EQU/112388/2009 (COMPETE-01-0124-FEDER-015247), and contracts Pest-C/EME/UI0285/2013 and Pest-C/EQB/UI0102/2013, both financed by FCT/MCTES (PIDDAC) and co-financed by the European Regional Development Fund through the program COMPETE.

\section{REFERENCES}

[1] Torczynski, J.R., O’Hern, T.J, Adkins, D.R., Jackson, N.B. \& Shollenberger, K.A., Advanced tomographic flow diagnostics for opaque multiphase fluids, sandia report SAND97-1176, UC-406, Sandia National Laboratories, Albuquerque, New Mexico, USA, 1997.

[2] Rasteiro, M.G., Silva, R., Garcia, F.A.P. \& Faia, P., Electrical tomography: a review of configurations and applications to particulate processes. KONA Powder and Particle Journal, 29, pp. 67-80, 2011.

http://dx.doi.org/10.14356/kona.2011010

[3] Nakagawa, M., Altobelli, S.A., Caprihan, A., Fukushima, E. \& Jeong, E.K., Noninvasive measurements of granular flows by magnetic resonance imaging. Experiments in Fluids, 16, pp. 54-60, 1993.

http://dx.doi.org/10.1007/BF00188507

[4] Sederman, A.J., Gladden, L.F. \& Mantle, M.D., Application of magnetic resonance imaging techniques to particulate systems. Advanced Powder Technology, 18, pp. 23-38, 2007. http://dx.doi.org/10.1163/156855207779768232

[5] Müller, C.R., Holland, D.J., Sederman, A.J., Mantle, M.D., Gladden, L.F. \& Davidson, J.F., Magnetic resonance imaging of fluidized beds. Powder Technology, 183, pp. 53-62, 2008.

http://dx.doi.org/10.1016/j.powtec.2007.11.029

[6] Dyakowski, T., Jeanmeure, L.F.C. \& Jaworski, A.J., Applications of electrical tomography for gas-solids and liquid-solids flows - a review. Powder Technology, 112, pp. 174-192, 2000. http://dx.doi.org/10.1016/S0032-5910(00)00292-8

[7] Xie, C.G., Reinecke, N., Beck, M.S., Mewes, D. \& Williams, R.A., Electrical tomography techniques for process engineering applications. The Chemical Engineering Journal and the Biochemical Engineering Journal, 56, pp. 127-133, 1995. http://dx.doi.org/10.1016/0923-0467(94)02907-5

[8] Takeda, Y., Velocity profile measurement by ultrasonic doppler method. Experimental Thermal and Fluid Science, 10, pp. 444-453, 1995. http://dx.doi.org/10.1016/0894-1777(94)00124-Q

[9] Wiklund, J.A., Stading, M., Pettersson, A.J. \& Rasmuson, A., A comparative study of UVP and LDA techniques for pulp suspensions in pipe flow. American Institute of Chemical Engineers Journal, 52, pp. 484-495, 2006. http://dx.doi.org/10.1002/aic.10653

[10] Ozaki, Y., Kawaguchi, T., Takeda, Y., Hishida, K. \& Maeda, M., High time resolution ultrasonic velocity profiler. Experimental Thermal and Fluid Science, 26, pp. 253-258, 2002. http://dx.doi.org/10.1016/S0894-1777(02)00134-6 
[11] Stevenson, R., Harrison, S.T.L., Mantle, M.D., Sederman, A.J., Moraczewski, T.L. \& Johns, M.L., Analysis of partial suspension in stirred mixing cells using both MRI and ERT. Chemical Engineering Science, 65, pp. 1385-1393, 2010. http://dx.doi.org/10.1016/j.ces.2009.10.006

[12] Chandrasekera, T.C., Wang, A., Holland, D.J., Marashdeh, Q., Pore, M., Wang, F., Sederman, A.J., Fan, L.S., Gladden, L.F. \& Dennis, J.S., A comparison of magnetic resonance imaging and electrical capacitance tomography: an air jet through a bed of particles. Powder Technology, 227, pp. 86-95, 2012.

http://dx.doi.org/10.1016/j.powtec.2012.03.005

[13] Faia, P.M., Ferreira, A.R., Santos, M.J., Santos, J.B., Silva, R., Rasteiro, M.G. \& Garcia, F.A.P., Imaging particulate two-phase flow in liquid suspensions with electric impedance tomography. Particulate Science and Technology, 30, pp. 329-342, 2012. http://dx.doi.org/10.1080/02726351.2011.575444

[14] Polydorides, N. \& Lionheart, W.R.B., A Matlab toolkit for three-dimensional electrical impedance tomography: a contribution to the electrical impedance and diffuse optical reconstruction software project. Measurement Science and Technology, 13(12), pp. 1871-1883, 2002.

http://dx.doi.org/10.1088/0957-0233/13/12/310

[15] Cheng, K.S., Isaacson, D., Newell, J.C. \& Gisser, D.G., Electrode models for electric current computed tomography. IEEE Transactions on Biomedical Engineering, 36(9), pp. 918-924, 1989.

http://dx.doi.org/10.1109/10.35300 